\title{
Conceptual design of a Charged Particle Beam Energy Spectrometer Utilizing Transition Radiation Grating
}

\author{
X.Z. Qiu, X.J. Wang, K. Batchelor and I. Ben-Zvi \\ National Synchrotron Light Source, Brookhaven National Laboratory \\ Upton, N.Y., 11973
}

\begin{abstract}
A new technique of measuring the energy spectrum of a charged particle beam using optical transition radiation is presented in this paper. The charged particle beam energy spectrum can be studied by analyzing the optical spectrum of the optical transition radiation produced in a multi-foil configuration. This type of energy spectrometer can have a very large energy acceptance which can not be matched by a simple conventional magnetic dipole spectrometer. The theory of transition radiation from a single boundary and from a foil with finite thickness are reviewed. Multi-foil transition radiation theory and its application for charged particle beam energy spectrum measurement are presented. The practical issues which might affect the performance of a multi-foil transition radiation energy spectrometer are discussed.
\end{abstract}

\section{Introduction}

A new charged particle beam energy spectrometer using transition radiation is presented in this paper. The spectrometer measures the electron beam energy spectrum by observing the interference effect of a multi-foil transition radiator. The energy acceptance of such a spectrometer can be extremely large.

We will review the basic physics for optical transition radiation from a single foil and a large number of parallel foils. With proper arrangement of the foil thickness and foil spacing, a strong interference can occur between radiation from different foils. The consequence will be the

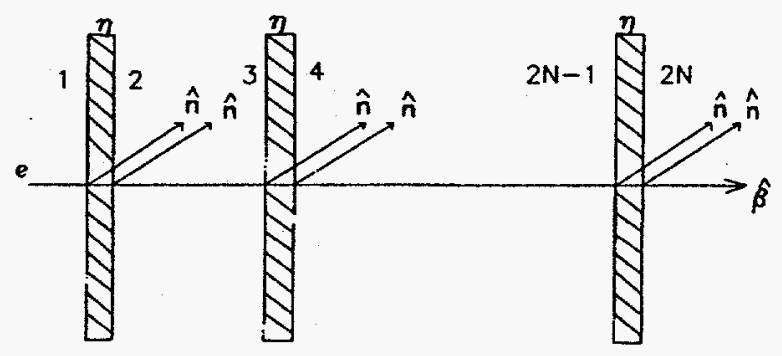

Figure 1: A charged particle travels through parallel foils. appearance of sharp interference peaks both in angular and spectral distributions. The angular and spectral locations of the these peaks critically depend on the energy and the incoming angle of the radiating particle. The angular spread and spectral width of the OTR reflect the energy and angular distribution of the charged particle beams. This property of the OTR can be used for charge particle beam energy spectrum measurement.

Finally, the major factors that may affect the performance of the energy spectrometer discussed.

Single-foil and multi-foil transition radiation

A relativistic particle of charge $e$ traveling in vacuum is entering foils with index of refraction $\eta$, as shown in figure 1. To simplify our discussion and illustrate the interesting physics, we make the following assumptions. We assume that $|\eta-1| \gg \gamma^{-2}$. The foils considered are produced so thin that the absorption of radiation in the foil can be ignored. Finally, we assume the particle is so energetic that the change in the particle trajectory due to Coulomb scattering is negligible.

When the particle crosses interface 1 , transition radiation will be produced in both forward and backward direction. We are only interested in the forward radiation. For $\theta \ll 1$, the intensity is given by, [1]

$$
\frac{d^{2} I_{1}}{d \omega d \Omega}=\frac{e^{2}}{\pi^{2} c} \frac{\theta^{2}}{\left(\gamma^{-2}+\theta^{2}\right)^{2}}
$$

where $\theta$ is the angle between the direction the particle velocity and the observation direction, $\gamma$ is the Lorentz factor.

After the particle has passed through the foil and crosses the interface 2 , it will again produce transition radiation in the forward direction. The magnitude of the radiation is the same as that produced at interface 1 , but is $180^{\circ}$ out of phase, The combined intensity is then,

$$
\begin{aligned}
\frac{d I_{12}^{2}}{d \omega d \Omega} & =\frac{e^{2}}{\pi^{2} c} \frac{\theta^{2}}{\left(\gamma^{-2}+\theta^{2}\right)^{2}} \\
& \times 4 \sin ^{2}\left\{\frac{\pi L_{12}}{2 \lambda}\left[\eta\left(\gamma^{-2}+\theta^{2}\right)+2(1-\eta)\right]\right\}
\end{aligned}
$$

This manuscript has been authored under contract number DE-AC02-76CH00016 with the U.S. Department of Energy. Accordingly, the U.S. Government retains a non-exclusive, royalty-free license to publish or reproduce the published form of this contribution, or allow others to do so, for U.S. Government purposes. 
Table I: Particles energy and apectrometer geometry

\begin{tabular}{|c|c|}
\hline$\gamma$ & $94,96,98,100,102$ \\
\hline & $104,106,108,110,112$ \\
\hline$N$ & 100 \\
\hline$\theta_{0}$ & $0.01 \mathrm{rad}$ \\
\hline$\eta$ & 1.5 \\
\hline$L_{12}$ & $100 \mathrm{~nm}$ \\
\hline$L_{23}$ & $10 \mathrm{~mm}$ \\
\hline$K_{c}$ & 2 \\
\hline
\end{tabular}

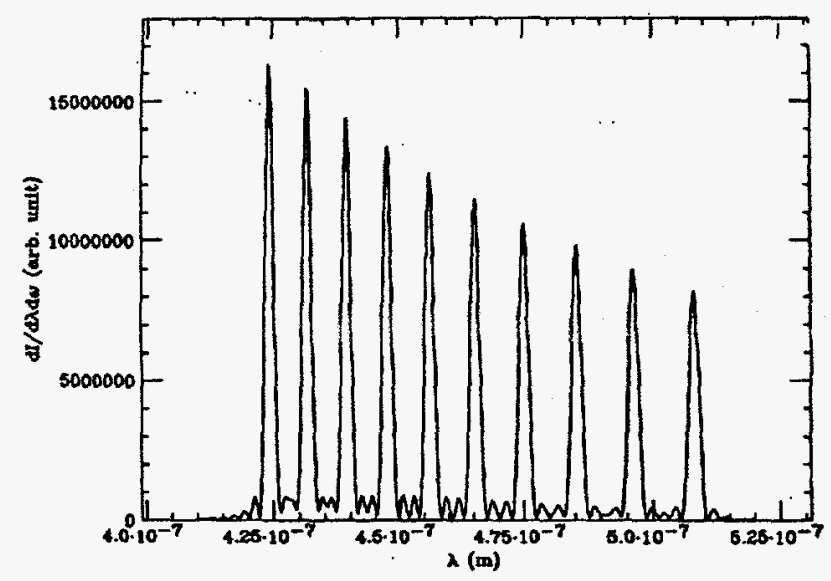

Figure 2: OTR spectrum for 10 particles with different energy

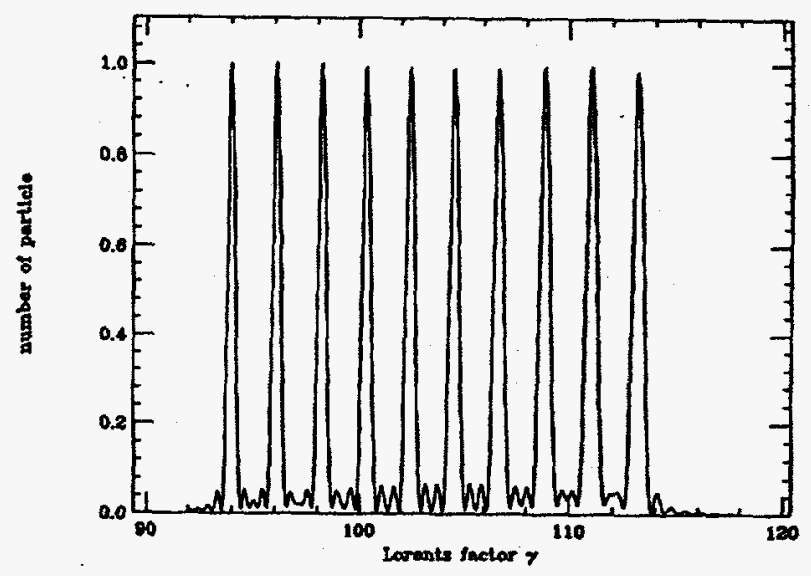

Figure 3: Normalized particle energy spectrum.

where $L_{12}$ is the thickness of the foil.

As the particle continues to travel through the foils. The total forward radiation is just the sum of the forward radiation from each foil,

$$
\frac{d^{2} I}{d \omega d \Omega}=\frac{d^{2} I_{12}}{d \omega d \Omega} \times \frac{\sin ^{2}\left(N \phi_{13} / 2\right)}{\sin ^{2}\left(\phi_{13} / 2\right)}
$$

where $N$ is the number of foils and $\phi_{13}$ is the phase delay of the radiation from interface 3 relative to that from interface 1 , is given by

$$
\begin{aligned}
\phi_{13} & =\frac{\pi L_{12}}{\lambda}\left[\eta\left(\gamma^{-2}+\theta^{2}\right)+2(1-\eta)\right] \\
& +\frac{\pi L_{23}}{\lambda}\left(\gamma^{-2}+\theta^{2}\right)
\end{aligned}
$$

The total intensity per unit frequency per unit solid angle can be written as, In analogy to an optical grating, if the conditions

$$
\begin{aligned}
\phi_{13} & =2 K_{\imath} \pi, \quad K_{\bullet}=\text { integer } \\
N & \gg 1
\end{aligned}
$$

are satisfied, sharp interference peaks will appear in the angular distribution for a particular wavelength of radiation and in the radiation spectrum for a particular observation angle. The spectral or angular location of these peaks can be used to determine the energy and incident angle of the radiating particle. We will concentrate on using the spectrum to determine the particle energy at a fixed observation angle.

As an example the transition radiation spectrum produced by 10 particles with the parameters given in Table Tb.example@ is plotted in Fig.2.

Normalization taking into account of the dependence of intensity on energy and the nonlinear relationship between wavelength and energy has to perform in order to recover the energy spectrum. The recovered energy spectrum is given in Fig.3.

The finite width of the peaks due to the finite number of foils will contribute to the uncertainty in energy. The uncertainty in energy determination due to the finite width of the peak is inversely proportional to the number of foils, and given by,

$$
\frac{\delta \gamma_{e}}{\gamma_{e}}=\frac{1}{N} \times \frac{\gamma_{e}^{2} \lambda_{e}}{L_{23}}
$$

In order to achieve good resolution in the energy determination, large number of foils are used. It is difficult for the radiation from the front and end foils to interfere if a large observation angle is used. This makes it necessary to observe the radiation at a smaller angle, even at a zero degree observation angle. For a perfect parallel charged particle beam, no radiation would be observed at this angle. However, the divergence of the beam and scattering of the particles in the foils will cause radiation in this direction.

The energy acceptance of a TRG energy spectrometer is quite large. In the case of a zero degree observation angle, if very thin foils are used, an energy acceptance of $\gamma \rightarrow 1.4 \gamma$, which is about $\pm 20 \%$, would be achievable. If relative thicker foils are used, acceptance of $\gamma \rightarrow 2 \gamma$, which is about $\pm 50 \%$, or higher should be achieved. 


\section{DISCLAIMER}

\section{Portions of this document may be illegible in electronic image products. Images are produced from the best available original document.}


Pratical issues concerning a TRG spectrometer

There are many factors may affect the performance of TRG spectrometer. We will discuss some of them in this section.

In order to maintain the desired energy resolution, the spread of interference peak due to the spread in observation angle should be smaller than the uncertainty in energy measurement. For observation angle $\theta_{0}$ other than zero degree, we must have

$$
\sigma_{z^{\prime}}<\frac{1}{\gamma_{e}} \times \frac{\delta \gamma_{e}}{\gamma_{e}} \times \frac{1}{\theta_{0} \gamma_{e}}
$$

where $\gamma_{e}$ is the Lorents factor of the particle under measurement, $\sigma_{z^{\prime}}$ is the rms beam divergence projected in the plane of observation. For the geometry in the example, $\sigma_{x^{\prime}}$ must be smaller than $100 \mu \mathrm{rad}$ for $1 \%$ energy measurement resolution.

In order to relax the tolerance of the measurement due to beam divergence, one can use a small observation angle. When $\theta_{0}=0^{\circ}$, the spread in energy measurement due to finite beam divergence is given by the curve,

$$
I\left(\gamma, \gamma_{e}\right) \propto \gamma_{e}^{4} \sigma_{z^{2}}^{2} \times u e^{-u}
$$

where

$$
u=\frac{\gamma-\gamma_{\varepsilon}}{\gamma_{\varepsilon}^{3} \sigma_{z^{\prime}}^{2}}, \quad 0<u<\infty
$$

The curve has its maximum at $u=1$. Therefore there will be a systematic shift of $\delta \gamma=\gamma_{e}^{3} \sigma_{z^{\prime}}^{2}$ in the energy spectrum measurement, which has to be corrected in the calibration. Notice that the signal is zero when $\sigma_{x^{\prime}}$ is gero. (9) has a width of about $\delta u \approx 1$, so the divergence of the beam must satisfy,

$$
\sigma_{x^{\prime}}<\frac{1}{\gamma_{e}} \times \sqrt{\frac{\delta \gamma_{e}}{\gamma_{e}}}
$$

Note the square root dependence on the desired energy resolution. For the geometry given in the example, if $K_{c}=1$ is used, the upper limit of $\sigma_{z^{\prime}}$ is about $1 \mathrm{mrad}$ for $1 \%$ resolution.

The total rms scattering angle of the particle in the foils and the rms angular alignment error of the foils should also satisfy (8) or (11). In the case of estimating scattering, special care has to be taken when designing a TRG spectrometer for an electron beam. Because the foils will be used in a TRG spectrometer are very thin, the Highland form of the emperical formula for multiple scattering tends to overestimate the scattering effect. Using the formula given by Lynch and Danl, [2] we eatimate the rms scattering angle for a $50 \mathrm{MeV}$ electron beam passes through 100 pieces of 100-nm-thick Carbon foils is about $1.13 \mathrm{mrad}$, which is comparable to the requirement for achieving $1 \%$ resolution.

When application of TRG spectrometer for heavy particle beams, like proton beams, the scattering is about three order of magnitudes smaller than electron beams with the same $\gamma$ factor. So in these cases, scattering can be ignored.

The foil thickness and spacing tolerance is given by,

$$
\begin{aligned}
\delta L_{23} & <\frac{2 \gamma_{e}^{2} \lambda_{e}}{N} \\
\delta L_{12} & <\frac{\lambda_{e}}{|1-\eta| N}
\end{aligned}
$$

For the geometry given in the example, we must have,

$$
\begin{aligned}
\delta L_{23} & <100 \mu m \\
\frac{\delta L_{12}}{L_{12}} & <10 \%
\end{aligned}
$$

conclusion

We have discussed the basic operational principle for a TRG spectrometer. By proper selection of foil thickness and spacing, energy acceptance on the order of 50 percent or larger with resonable resolution can easily be achieved. We have also considered major factors may affect the performance of the energy spectrometer.

\section{Acknowledgements}

The authors wish to thank Dr. R. Fernow and Mr. S. Ulc for providing useful information about thin foils, and the entire ATF staff for their continuous support.

\section{References}

[1] L. Wartski, S. Roland, J. Lasalle, M. Bolore, J. Appl. Phys. 46, 3644 (1975).

[2] G. Lynch and O. Danl, Nucl. Instr. And Meth. B58, 6 (1991).

\section{DISCLAIMER}

This report was prepared as an account of work sponsored by an agency of the United States Government. Neither the United States Government nor any agency thereof, nor any of their employees, makes any warranty, express or implied, or assumes any legal liability or responsibility for the accuracy, completeness, or usefulness of any information, apparatus, product, or process disclosed, or represents that its use would not infringe privately owned rights. Reference herein to any specific commercial product, process, or service by trade name, trademark, manufacturer, or otherwise does not necessarily constitute or imply its endorsement, recommendation, or favoring by the United States Government or any agency thereof. The views and opinions of authors expressed herein do not necessarily state or reflect those of the United States Government or any agency thereof. 